\title{
Article
}

\section{The Volatile Phytochemistry of Monarda Species Growing in South Alabama}

\author{
Sims K. Lawson ${ }^{1}$, Prabodh Satyal ${ }^{2}$ and William N. Setzer $2,3, * \mathbb{D}$ \\ Kirkland Gardens, P.O. Box 176, Newville, AL 36353, USA; skirkland.lawson@ufl.edu \\ Aromatic Plant Research Center, 230 N 1200 E, Suite 100, Lehi, UT 84043, USA; psatyal@aromaticplant.org \\ Department of Chemistry, University of Alabama in Huntsville, Huntsville, AL 35899, USA \\ *orrespondence: wsetzer@chemistry.uah.edu; Tel.: +1-256-824-6519
}

check for updates

Citation: Lawson, S.K.; Satyal, P.; Setzer, W.N. The Volatile

Phytochemistry of Monarda Species Growing in South Alabama. Plants 2021, 10, 482. https://doi.org/ $10.3390 /$ plants10030482

Academic Editors: Jésus Palá-Pául, Joseph Brophy and Laura Cornara

Received: 9 February 2021

Accepted: 26 February 2021

Published: 4 March 2021

Publisher's Note: MDPI stays neutral with regard to jurisdictional claims in published maps and institutional affiliations.

Copyright: (c) 2021 by the authors. Licensee MDPI, Basel, Switzerland. This article is an open access article distributed under the terms and conditions of the Creative Commons Attribution (CC BY) license (https:/ / creativecommons.org/licenses/by/ $4.0 /)$.

\begin{abstract}
The genus Monarda (family Lamiaceae) contains 22 species of which three are native to southern Alabama, M. citriodora, M. fistulosa, and M. punctata. Several species of Monarda have been used in traditional medicines of Native Americans, and this present study is part of an ongoing project to add to our understanding of Native American pharmacopeia. Plant material from M. citriodora, M. fistulosa, and M. punctata was collected in south Alabama and the essential oils obtained by hydrodistillation. The essential oils were analyzed by gas chromatographic techniques to determine the chemical compositions as well as enantiomeric distributions. The compounds thymol, carvacrol, $p$-cymene, and their derivatives were the primary terpenoid components found in the essential oils. The known biological activities of these compounds are consistent with the traditional uses of Monarda species to treat wounds, skin infections, colds, and fevers.
\end{abstract}

Keywords: Monarda citriodora; Monarda fistulosa; Monarda punctata; essential oil; thymol; carvacrol; p-cymene

\section{Introduction}

The Plant List [1] shows 22 different Monarda L. (Lamiaceae) species, 18 of which occur in the United States [2]. There are three Monarda species native to south Alabama, namely Monarda citriodora Cerv. ex Lag., Monarda fistulosa L., and Monarda punctata L. (see Figure 1) [2].

Several Monarda species have been used by Native Americans as medicinal plants [3]. For example, $M$. fistulosa was used by the Blackfoot, Navajo, Lakota, and Winnebago people to treat boils, cuts and wounds; the Cherokee, Chippewa, Flathead, Ojibwa, and Tewa used the plant to treat colds, fever, and influenza; the Crow, Lakota, Menominee, and Ojibwa used the plant for coughs, catarrh, and other respiratory problems. Monarda punctata was used by the Delaware, Mohegan Nanticoke, and Navajo tribes to treat colds, fever coughs, and catarrh.

Both $M$. citriodora and M. fistulosa are popular ornamentals and have been introduced to temperate locations around the world [4-6]. Geographical location likely plays an important role in the phytochemistry of Monarda species. To our knowledge, however, there have been no previous examinations of $M$. citriodora, M. fistulosa, or M. punctata growing in their native range of south Alabama. In this work, we have examined the chemical compositions and enantiomeric distributions of essential oils of the three Monarda species from south Alabama. 


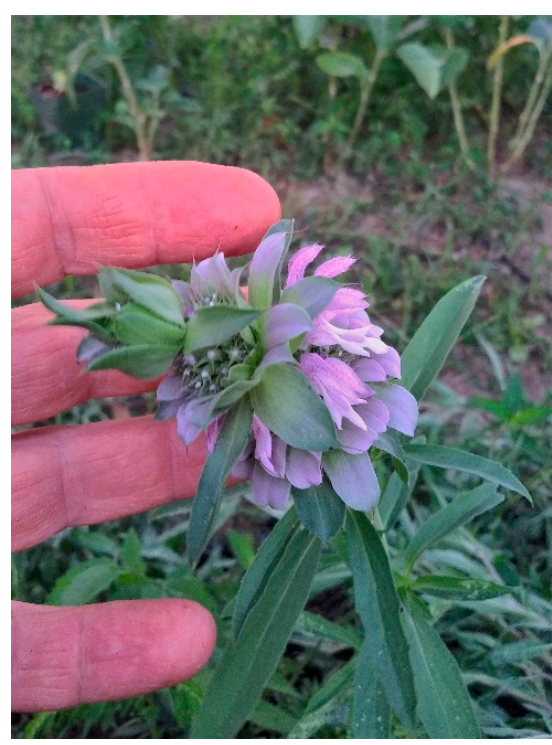

Monarda citriodora

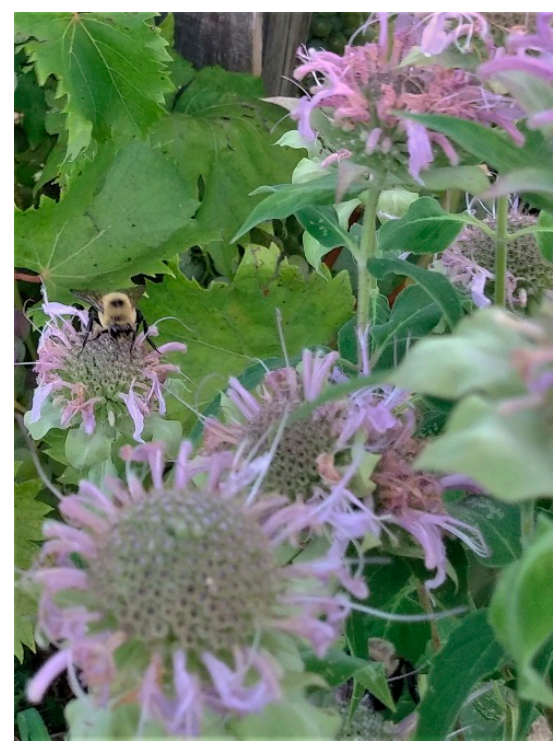

Monarda fistulosa

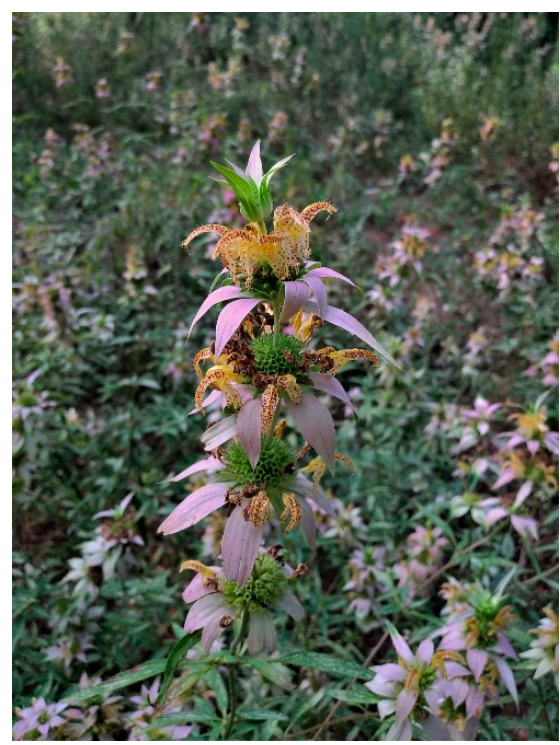

Monarda punctata

Figure 1. Monarda species discussed in this work (photographs by S. K. L).

\section{Results}

\subsection{Monarda Citriodora}

The $M$. citriodora essential oils were obtained as clear orange oils. The essential oil yields for $M$. citriodora aerial parts essential oil were $1.59 \%$ and $1.79 \%$ for samples \#1 and \#2, respectively, while the root essential oil was obtained in $0.879 \%$ yield. The chemical compositions of the essential oils from the aerial parts and the roots of $M$. citriodora cultivated in south Alabama are summarized in Table 1. The essential oils were dominated by the phenolic monoterpenoids thymol $\left(R I_{d b}=1289\right)$ and carvacrol $\left(R I_{d b}=1296\right)$. The other major components were $p$-cymene $\left(\mathrm{RI}_{\mathrm{db}}=1024\right)$ and thymol methyl ether $\left(\mathrm{RI}_{\mathrm{db}}=1239\right)$.

Table 1. Essential oil compositions of Monarda citriodora cultivated in south Alabama.

\begin{tabular}{|c|c|c|c|c|c|c|c|c|}
\hline \multirow[b]{2}{*}{$\mathbf{R I}_{\text {calc }}$} & \multirow[b]{2}{*}{$\mathbf{R I}_{\mathrm{db}}$} & \multirow[b]{2}{*}{ Compound } & \multicolumn{4}{|c|}{ Aerial Parts Essential Oil } & \multicolumn{2}{|c|}{ Root Essential Oil } \\
\hline & & & $\# 1, \%$ & ED, (+):(-) & $\# 2, \%$ & ED, $(+):(-)$ & $\# 2, \%$ & ED, (+):(-) \\
\hline 923 & 925 & $\alpha$-Thujene & 1.0 & 69.0:31.0 & 0.8 & $66.6: 33.4$ & 0.5 & $64.2: 35.8$ \\
\hline 930 & 932 & $\alpha$-Pinene & 0.3 & $84.2: 15.8$ & 0.3 & $62.8: 37.2$ & 0.2 & $74.5: 25.5$ \\
\hline 947 & 950 & Camphene & $\operatorname{tr}$ & & $\operatorname{tr}$ & & $\operatorname{tr}$ & \\
\hline 970 & 971 & Sabinene & $\operatorname{tr}$ & & $\operatorname{tr}$ & & - & \\
\hline 975 & 978 & $\beta$-Pinene & 0.1 & $64.5: 35.5$ & 0.1 & & 0.1 & \\
\hline 976 & 974 & 1-Octen-3-ol & 0.7 & & 0.7 & & 0.5 & \\
\hline 983 & 984 & 3-Octanone & 0.1 & & 0.2 & & 0.3 & \\
\hline 987 & 989 & Myrcene & 0.7 & & 0.4 & & 0.3 & \\
\hline 995 & 996 & 3-Octanol & 0.2 & & 0.3 & & 0.3 & \\
\hline 1002 & 1004 & Octanal & $\operatorname{tr}$ & & $\operatorname{tr}$ & & - & \\
\hline 1003 & 1004 & $p$-Mentha-1(7),8-diene & $\operatorname{tr}$ & & - & & - & \\
\hline 1005 & 1006 & $\alpha$-Phellandrene & 0.1 & 95.1:4.9 & 0.1 & 100:0 & $\operatorname{tr}$ & 100:0 \\
\hline 1007 & 1008 & $\delta$-3-Carene & 0.1 & $100: 0$ & 0.1 & 100:0 & 0.1 & $100: 0$ \\
\hline 1015 & 1017 & $\alpha$-Terpinene & 1.8 & $100: 0$ & 1.1 & $100: 0$ & 0.7 & $100: 0$ \\
\hline 1016 & 1022 & $m$-Cymene & $\operatorname{tr}$ & & $\operatorname{tr}$ & & - & \\
\hline 1023 & 1024 & p-Cymene & 7.8 & & 6.4 & & 7.2 & \\
\hline 1027 & 1030 & Limonene & 0.5 & $26.7: 73.3$ & 0.4 & 63.3:36.7 & 0.3 & 57.3:42.7 \\
\hline 1028 & 1029 & $\beta$-Phellandrene & 0.2 & $0: 100$ & 0.1 & $0: 100$ & 0.1 & $0: 100$ \\
\hline 1030 & 1033 & Benzyl alcohol & - & & - & & 0.4 & \\
\hline 1030 & 1030 & 1,8-Cineole & 0.2 & & 0.3 & & 0.2 & \\
\hline 1055 & 1057 & $\gamma$-Terpinene & 1.7 & & 0.5 & & 0.2 & \\
\hline 1067 & 1069 & cis-Sabinene hydrate & 0.8 & 95.3:4.7 & 1.0 & $89.5: 10.5$ & 0.9 & $90.7: 9.3$ \\
\hline
\end{tabular}


Table 1. Cont.

\begin{tabular}{|c|c|c|c|c|c|c|c|c|}
\hline \multirow[b]{2}{*}{$\mathrm{RI}_{\text {calc }}$} & \multirow[b]{2}{*}{$\mathrm{RI}_{\mathrm{db}}$} & \multirow[b]{2}{*}{ Compound } & \multicolumn{4}{|c|}{ Aerial Parts Essential Oil } & \multicolumn{2}{|c|}{ Root Essential Oil } \\
\hline & & & $\# 1, \%$ & $\mathrm{ED},(+):(-)$ & $\# 2, \%$ & ED, (+):(-) & $\# 2, \%$ & $\mathrm{ED},(+):(-)$ \\
\hline 1083 & 1086 & Terpinolene & $\operatorname{tr}$ & & $\operatorname{tr}$ & & $\operatorname{tr}$ & \\
\hline 1084 & 1086 & $\begin{array}{l}\text { trans-Linalool oxide } \\
\text { (furanoid) }\end{array}$ & - & & $\operatorname{tr}$ & & 0.1 & \\
\hline 1087 & 1093 & p-Cymenene & - & & - & & $\operatorname{tr}$ & \\
\hline 1098 & 1099 & Linalool & 0.1 & 71.9:28.1 & 0.1 & $49.7: 50.3$ & 0.3 & 50.1:49.9 \\
\hline 1099 & 1099 & trans-Sabinene hydrate & $\operatorname{tr}$ & $76.5: 23.5$ & 0.3 & 71.1:28.9 & 0.3 & $66.8: 33.2$ \\
\hline 1165 & 1167 & exo-Acetoxycamphene & - & & - & & 0.1 & \\
\hline 1169 & 1170 & Borneol & 0.1 & $0: 100$ & 0.1 & $0: 100$ & 0.3 & $0: 100$ \\
\hline 1178 & 1180 & Terpinen-4-ol & 0.4 & $60.2: 39.8$ & 0.4 & $58.5: 41.5$ & 1.9 & 20.9:79.1 \\
\hline 1183 & 1186 & p-Cymen-8-ol & - & & - & & 0.1 & \\
\hline 1187 & 1190 & Methyl salicylate & $\operatorname{tr}$ & & - & & - & \\
\hline 1195 & 1195 & $\alpha$-Terpineol & 0.1 & 100:0 & 0.1 & $100: 0$ & 0.2 & 100:0 \\
\hline 1196 & 1197 & Methyl chavicol (= Estragole) & - & & 1.5 & & - & \\
\hline 1236 & 1239 & Thymol methyl ether & 4.4 & & 5.6 & & 11.3 & \\
\hline 1252 & 1252 & Thymoquinone & 0.2 & & 0.7 & & 1.3 & \\
\hline 1253 & 1246 & Carvone & $\operatorname{tr}$ & 39.9:60.1 & - & & - & \\
\hline 1290 & 1289 & Thymol & 38.2 & & 37.0 & & 29.0 & \\
\hline 1297 & 1296 & Carvacrol & 38.3 & & 39.9 & & 38.3 & \\
\hline 1305 & 1309 & 4-Vinylguaiacol & - & & - & & 0.1 & \\
\hline 1306 & 1306 & iso-Ascaridole & $\operatorname{tr}$ & & $\operatorname{tr}$ & & - & \\
\hline 1342 & 1345 & Thymyl acetate & 0.3 & & 0.2 & & 0.3 & \\
\hline 1347 & 1356 & Eugenol & $\operatorname{tr}$ & & - & & 0.3 & \\
\hline 1361 & 1365 & Carvacryl acetate & 0.8 & & 0.5 & & 1.0 & \\
\hline 1372 & 1375 & $\alpha$-Copaene & $\operatorname{tr}$ & $100: 0$ & $\operatorname{tr}$ & & 0.1 & $100: 0$ \\
\hline 1380 & 1382 & $\beta$-Bourbonene & $\operatorname{tr}$ & & $\operatorname{tr}$ & & 0.1 & \\
\hline 1389 & 1392 & (Z)-Jasmone & $\operatorname{tr}$ & & $\operatorname{tr}$ & & $\operatorname{tr}$ & \\
\hline 1398 & 1398 & Cyperene & - & & - & & 0.2 & \\
\hline 1404 & 1408 & Decyl acetate & $\operatorname{tr}$ & & - & & - & \\
\hline 1415 & 1417 & $(E)-\beta$-Caryophyllene & 0.3 & 100:0 & 0.4 & 100:0 & 0.5 & 100:0 \\
\hline 1426 & 1430 & $\beta$-Copaene & $\operatorname{tr}$ & & $\operatorname{tr}$ & & $\operatorname{tr}$ & \\
\hline 1451 & 1453 & $\alpha$-Humulene & $\operatorname{tr}$ & & $\operatorname{tr}$ & & $\operatorname{tr}$ & \\
\hline 1457 & 1457 & Rotundene & - & & - & & 0.1 & \\
\hline 1471 & 1475 & $\gamma$-Muurolene & $\operatorname{tr}$ & & 0.1 & & 0.1 & \\
\hline 1473 & 1481 & $(E)$ - $\beta$-Ionone & - & & - & & $\operatorname{tr}$ & \\
\hline 1477 & 1480 & Germacrene D & 0.1 & $100: 0$ & 0.1 & $100: 0$ & 0.1 & \\
\hline 1481 & 1485 & $\gamma$-Thujaplicin & $\operatorname{tr}$ & & - & & 0.1 & \\
\hline 1483 & 1489 & $\beta$-Selinene & $\operatorname{tr}$ & & - & & - & \\
\hline 1487 & 1490 & $\gamma$-Amorphene & $\operatorname{tr}$ & & - & & - & \\
\hline 1491 & 1497 & $\alpha$-Selinene & $\operatorname{tr}$ & & $\operatorname{tr}$ & & 0.1 & \\
\hline 1494 & 1497 & $\alpha$-Muurolene & $\operatorname{tr}$ & & $\operatorname{tr}$ & & $\operatorname{tr}$ & \\
\hline 1509 & 1512 & $\gamma$-Cadinene & $\operatorname{tr}$ & & $\operatorname{tr}$ & & 0.1 & \\
\hline 1514 & 1518 & $\delta$-Cadinene & 0.1 & & 0.1 & & 0.1 & $0: 100$ \\
\hline 1548 & 1549 & Thymohydroquinone & 0.3 & & 0.1 & & 0.1 & \\
\hline 1577 & 1577 & Caryophyllene oxide & $\operatorname{tr}$ & & 0.1 & & 0.1 & \\
\hline 1649 & 1655 & $\alpha$-Cadinol & - & & - & & 0.1 & \\
\hline 1689 & 1691 & Cyperotundone & - & & - & & 0.2 & \\
\hline \multirow[t]{8}{*}{1835} & 1841 & Phytone & - & & - & & 0.1 & \\
\hline & & Monoterpene hydrocarbons & 14.3 & & 10.2 & & 9.6 & \\
\hline & & Oxygenated monoterpenoids & 84.0 & & 86.3 & & 86.0 & \\
\hline & & Sesquiterpene hydrocarbons & 0.5 & & 0.7 & & 1.4 & \\
\hline & & Oxygenated sesquiterpenoids & $\operatorname{tr}$ & & 0.1 & & 0.4 & \\
\hline & & Benzenoid aromatics & $\operatorname{tr}$ & & 1.5 & & 0.8 & \\
\hline & & Others & 1.0 & & 1.2 & & 1.2 & \\
\hline & & Total identified & 99.8 & & 99.8 & & 99.3 & \\
\hline
\end{tabular}

$\mathrm{RI}_{\text {calc }}=$ Retention indices determined with respect to a homologous series of $n$-alkanes on a ZB-5ms column. $\mathrm{RI}_{\mathrm{db}}=$ Retention indices from the databases [7-10]. \#1 = Plant sample \#1. \#2 = Plant sample \#2. $-=$ Not observed. ED = Enantiomeric distribution (dextrorotatory enantiomer: levorotatory enantiomer). $\operatorname{tr}=\operatorname{Trace}(<0.05 \%)$. 
Chiral gas chromatography-mass spectrometry (GC-MS) analysis of the M. citriodora essential oils revealed the (+)-enantiomers to be the major stereoisomers for $\alpha$-thujene, $\alpha$-pinene, $\beta$-pinene, $\alpha$-phellandrene, $\delta$-3-carene, $\alpha$-terpinene, cis-sabinene hydrate, transsabinene hydrate, $\alpha$-terpineol, $\alpha$-copaene, $(E)-\beta$-caryophyllene, and germacrene $\mathrm{D}$. On the other hand, the (-)-enantiomer was dominant for $\beta$-phellandrene, borneol, carvone, and $\delta$ cadinene. Limonene showed variation in the enantiomeric distributions with (+)-limonene in $26.7 \%, 63.3 \%$, and $57.3 \%$ for aerial parts $\# 1, \# 2$, and roots essential oils, respectively. Likewise, linalool also showed variation with (+)-linalool of $71.9 \%, 49.7 \%$, and $50.1 \%$. $(+)$-Terpinen-4-ol was the predominant enantiomer in the aerial parts essential oils $(60.2 \%$ and $58.5 \%)$, but (-)-terpinen-4-ol (79.1\%) was dominant in the root essential oil.

\subsection{Monarda Fistulosa}

Monarda fistulosa essential oils were obtained in $2.66-4.83 \%$ yields as bright orange oils. The chemical compositions of the essential oils from the aerial parts of $M$. fistulosa are summarized in Table 2. In samples \#1 and \#2, thymol $\left(\mathrm{RI}_{\mathrm{db}}=1289\right)$ dominated the compositions $(54.3 \%$ and $62.2 \%$, respectively) with lesser quantities of $p$-cymene $\left(\mathrm{RI}_{\mathrm{db}}=1024,12.1 \%\right.$ and $\left.10.2 \%\right)$, limonene $\left(\mathrm{RI}_{\mathrm{db}}=1030,6.1 \%\right.$ and $\left.3.7 \%\right)$, carvacrol $\left(\mathrm{RI}_{\mathrm{db}}=1296,5.9 \%\right.$ and $\left.6.6 \%\right)$, and thymoquinone $\left(\mathrm{RI}_{\mathrm{db}}=1252,8.4 \%\right.$ and $\left.2.3 \%\right)$. Curiously, sample \#3, although qualitatively similar, had a very different quantitative composition with thymoquinone as the most abundant constituent $(41.3 \%)$ followed by $p$-cymene $(21.9 \%)$, but with lower concentrations of thymol $(8.9 \%)$ and carvacrol $(1.6 \%)$.

Table 2. Chemical composition of Monarda fistulosa essential oils cultivated in south Alabama.

\begin{tabular}{|c|c|c|c|c|c|c|c|c|}
\hline $\mathbf{R I}_{\text {calc }}$ & $\mathbf{R I}_{\mathrm{db}}$ & Compound & $\# 1, \%$ & ED, (+):(-) & $\begin{array}{c}\text { Aerial Par } \\
\quad \# 2, \%\end{array}$ & $\begin{array}{l}\text { Essential Oil } \\
\text { ED, (+):(-) }\end{array}$ & $\# 3, \%$ & ED, $(+):(-)$ \\
\hline 923 & 925 & $\alpha$-Thujene & 1.2 & $72.5: 27.5$ & 0.8 & $72.8: 27.2$ & 0.9 & $71.2: 28.8$ \\
\hline 930 & 932 & $\alpha$-Pinene & 0.5 & 59.2:40.8 & 0.3 & $63.8: 36.2$ & 0.5 & 61.0:39.0 \\
\hline 947 & 950 & Camphene & 0.1 & 100:0 & 0.1 & 100:0 & 0.2 & 100:0 \\
\hline 971 & 971 & Sabinene & 0.2 & 58.4:41.6 & $\operatorname{tr}$ & & 0.2 & 59.0:41.0 \\
\hline 973 & 973 & 1-Octen-3-one & - & & - & & 0.1 & \\
\hline 975 & 978 & $\beta$-Pinene & 0.2 & 57.3:42.7 & - & & 0.2 & 57.9:42.1 \\
\hline 978 & 978 & 1-Octen-3-ol & 3.0 & & 3.3 & & 3.3 & \\
\hline 982 & 984 & 3-Octanone & $\operatorname{tr}$ & & 0.1 & & 0.1 & \\
\hline 987 & 989 & Myrcene & $\operatorname{tr}$ & & 0.3 & & 0.1 & \\
\hline 995 & 996 & 3-Octanol & $\operatorname{tr}$ & & 0.1 & & 0.1 & \\
\hline 1004 & 1004 & $p$-Mentha-1(7),8-diene & $\operatorname{tr}$ & & $\operatorname{tr}$ & & $\operatorname{tr}$ & \\
\hline 1006 & 1006 & $\alpha$-Phellandrene & 0.1 & $95.5: 4.5$ & 0.2 & $95.4: 4.6$ & 0.1 & $93.4: 6.6$ \\
\hline 1008 & 1008 & $\delta$-3-Carene & 0.1 & 100:0 & 0.1 & 100:0 & 0.1 & 100:0 \\
\hline 1016 & 1017 & $\alpha$-Terpinene & 2.1 & $100: 0$ & 2.3 & $100: 0$ & 0.8 & $100: 0$ \\
\hline 1019 & 1022 & m-Cymene & $\operatorname{tr}$ & & $\operatorname{tr}$ & & 0.1 & \\
\hline 1024 & 1024 & $p$-Cymene & 12.1 & & 10.2 & & 21.9 & \\
\hline 1025 & 1026 & 2-Acetyl-3-methylfuran & - & & - & & 0.5 & \\
\hline 1029 & 1030 & Limonene & 6.1 & $0.5: 99.5$ & 3.7 & 2.6:97.4 & 6.3 & $1.2: 98.8$ \\
\hline 1030 & 1031 & $\beta$-Phellandrene & 0.2 & $0: 100$ & 0.2 & $0: 100$ & 0.2 & $0: 100$ \\
\hline 1031 & 1030 & 1,8-Cineole & 0.1 & & 0.1 & & 0.1 & \\
\hline 1056 & 1057 & $\gamma$-Terpinene & $\operatorname{tr}$ & & 0.1 & & $\operatorname{tr}$ & \\
\hline 1069 & 1069 & cis-Sabinene hydrate & 1.2 & 95.8:4.2 & 1.3 & 96.3:3.7 & 2.4 & $96.5: 3.5$ \\
\hline 1078 & 1079 & 1-Nonen-3-ol & 0.1 & & 0.1 & & 0.1 & \\
\hline 1084 & 1086 & Terpinolene & $\operatorname{tr}$ & & 0.1 & & $\operatorname{tr}$ & \\
\hline 1089 & 1091 & $p$-Cymenene & $\operatorname{tr}$ & & $\operatorname{tr}$ & & 0.1 & \\
\hline 1098 & 1099 & Linalool & $\operatorname{tr}$ & $37.8: 62.2$ & $\operatorname{tr}$ & $37.5: 62.5$ & $\operatorname{tr}$ & $37.9: 62.1$ \\
\hline 1099 & 1099 & trans-Sabinene hydrate & 0.2 & $75.9: 24.1$ & 0.3 & $75.0: 25.0$ & 0.5 & $75.3: 24.7$ \\
\hline 1103 & 1107 & Nonanal & $\operatorname{tr}$ & & - & & $\operatorname{tr}$ & \\
\hline 1115 & 1112 & (E)-2,4-Dimethylhepta-2,4-dienal & - & & - & & 0.2 & \\
\hline 1121 & 1121 & trans- $p$-Mentha-2,8-dien-1-ol & $\operatorname{tr}$ & & $\operatorname{tr}$ & & 0.3 & \\
\hline 1123 & 1124 & cis-p-Menth-2-en-1-ol & $\operatorname{tr}$ & & $\operatorname{tr}$ & & $\operatorname{tr}$ & \\
\hline 1130 & 1132 & cis-Limonene oxide & $\operatorname{tr}$ & & - & & 0.1 & \\
\hline
\end{tabular}


Table 2. Cont.

\begin{tabular}{|c|c|c|c|c|c|c|c|c|}
\hline \multirow[b]{2}{*}{$\mathrm{RI}_{\text {calc }}$} & \multirow[b]{2}{*}{$\mathbf{R I}_{\mathrm{db}}$} & \multirow[b]{2}{*}{ Compound } & \multicolumn{6}{|c|}{ Aerial Parts Essential Oil } \\
\hline & & & $\# 1, \%$ & ED, (+):(-) & $\# 2, \%$ & ED, $(+):(-)$ & $\# 3, \%$ & ED, (+):(-) \\
\hline 1133 & 1135 & 2-Vinylanisole & 0.1 & & $\operatorname{tr}$ & & $\operatorname{tr}$ & \\
\hline 1134 & 1137 & cis- $p$-Mentha-2,8-dien-1-ol & - & & - & & 0.3 & \\
\hline 1135 & 1138 & trans-Limonene oxide & $\operatorname{tr}$ & & - & & - & \\
\hline 1137 & 1138 & trans-Sabinol & - & & - & & $\operatorname{tr}$ & \\
\hline 1138 & 1140 & trans-Pinocarveol & - & & - & & 0.1 & \\
\hline 1139 & 1141 & cis-Verbenol & - & & - & & $\operatorname{tr}$ & \\
\hline 1143 & 1145 & trans-Verbenol & - & & - & & 0.3 & \\
\hline 1144 & 1145 & Camphor & - & & - & & $\operatorname{tr}$ & \\
\hline 1160 & 1164 & Pinocarvone & - & & - & & $\operatorname{tr}$ & \\
\hline 1161 & 1162 & (Z)-iso-Citral & - & & - & & $\operatorname{tr}$ & \\
\hline 1167 & 1168 & trans-Phellandrene epoxide & - & & - & & 0.1 & \\
\hline 1170 & 1170 & Borneol & 0.5 & $0: 100$ & 0.2 & $0: 100$ & 0.7 & $0: 100$ \\
\hline 1179 & 1180 & Terpinen-4-ol & 0.4 & $63.3: 36.7$ & 0.5 & $63.2: 36.8$ & 0.5 & \\
\hline 1186 & 1186 & $p$-Cymen-8-ol & 0.1 & & $\operatorname{tr}$ & & 0.6 & \\
\hline 1195 & 1195 & $\alpha$-Terpineol & 0.3 & 100:0 & 0.2 & 100:0 & 0.3 & \\
\hline 1197 & 1198 & Methylchavicol (= Estragole) & - & & 0.1 & & 0.1 & \\
\hline 1197 & 1198 & cis-Piperitol & - & & - & & 0.2 & \\
\hline 1217 & 1218 & trans-Carveol & - & & - & & 0.2 & \\
\hline 1231 & 1232 & cis-Carveol & - & & - & & 0.1 & \\
\hline 1240 & 1242 & Cuminaldehyde & - & & - & & 0.1 & \\
\hline 1241 & 1242 & Carvone & - & & - & & 0.3 & \\
\hline 1250 & 1241 & Pulegone & - & & 0.2 & & - & \\
\hline 1252 & 1252 & Thymoquinone & 8.4 & & 2.3 & & 41.3 & \\
\hline 1281 & 1282 & Bornyl acetate & - & & - & & 0.1 & \\
\hline 1284 & 1286 & Cogeijerene & 0.1 & & - & & - & \\
\hline 1291 & 1291 & $p$-Cymen-7-ol & $\operatorname{tr}$ & & - & & 0.2 & \\
\hline 1295 & 1293 & Thymol & 54.3 & & 62.2 & & 8.9 & \\
\hline 1300 & 1300 & Carvacrol & 5.9 & & 6.6 & & 1.6 & \\
\hline 1307 & 1306 & iso-Ascaridole & $\operatorname{tr}$ & & $\operatorname{tr}$ & & 0.1 & \\
\hline 1345 & 1346 & $\alpha$-Cubebene & $\operatorname{tr}$ & & $\operatorname{tr}$ & & $\operatorname{tr}$ & \\
\hline 1351 & 1356 & Eugenol & $\operatorname{tr}$ & & $\operatorname{tr}$ & & - & \\
\hline 1373 & 1375 & $\alpha$-Copaene & 0.1 & $100: 0$ & 0.1 & $100: 0$ & 0.1 & $100: 0$ \\
\hline 1382 & 1382 & $\beta$-Bourbonene & 0.1 & & 0.1 & & 0.1 & \\
\hline 1387 & 1387 & trans- $\beta$-Elemene & $\operatorname{tr}$ & & $\operatorname{tr}$ & & 0.1 & \\
\hline 1418 & 1419 & $\beta$-Ylangene & & & $\operatorname{tr}$ & & 0.1 & \\
\hline 1419 & 1417 & $(E)$ - $\beta$-Caryophyllene & 0.3 & 100:0 & 0.3 & 100:0 & 0.2 & 100:0 \\
\hline 1427 & 1430 & $\beta$-Copaene & 0.1 & & 0.1 & & 0.1 & \\
\hline 1452 & 1453 & $\alpha$-Humulene & $\operatorname{tr}$ & & $\operatorname{tr}$ & & $\operatorname{tr}$ & \\
\hline 1473 & 1475 & $\gamma$-Muurolene & 0.1 & & 0.2 & & 0.1 & \\
\hline 1479 & 1479 & $\alpha$-Amorphene & - & & $\operatorname{tr}$ & & - & \\
\hline 1480 & 1483 & trans- $\beta$-Bergamotene & 0.1 & & - & & 0.1 & \\
\hline 1481 & 1480 & Germacrene D & 0.7 & $100: 0$ & 0.6 & $100: 0$ & 0.6 & $100: 0$ \\
\hline 1484 & 1485 & $\gamma$-Thujaplicin & 0.4 & & 0.2 & & 1.3 & \\
\hline 1488 & 1490 & $\gamma$-Amorphene & $\operatorname{tr}$ & & - & & - & \\
\hline 1491 & 1492 & $\beta$-Selinene & 0.1 & & 0.1 & & 0.1 & \\
\hline 1493 & 1492 & trans-Muurola-4(14),5-diene & - & & 0.1 & & - & \\
\hline 1496 & 1497 & epi-Cubebol & - & & $\operatorname{tr}$ & & - & \\
\hline 1497 & 1497 & $\alpha$-Selinene & - & & 0.1 & & - & \\
\hline 1498 & 1497 & $\alpha$-Muurolene & $\operatorname{tr}$ & & 0.1 & & $\operatorname{tr}$ & \\
\hline 1510 & 1512 & $\gamma$-Cadinene & 0.1 & & 0.2 & & 0.1 & \\
\hline 1511 & 1515 & Cubebol & $\operatorname{tr}$ & & - & & - & \\
\hline 1517 & 1518 & $\delta$-Cadinene & 0.1 & & 0.3 & & 0.1 & \\
\hline 1518 & 1519 & trans-Calamenene & $\operatorname{tr}$ & & $\operatorname{tr}$ & & - & \\
\hline 1520 & 1523 & $\beta$-Sesquiphellandrene & $\operatorname{tr}$ & & - & & - & \\
\hline 1537 & 1538 & $\alpha$-Cadinene & $\operatorname{tr}$ & & $\operatorname{tr}$ & & - & \\
\hline 1542 & 1541 & $\alpha$-Calacorene & $\operatorname{tr}$ & & $\operatorname{tr}$ & & - & \\
\hline 1543 & 1546 & $\alpha$-Elemol & $\operatorname{tr}$ & & - & & - & \\
\hline 1548 & 1554 & Thymohydroquinone & 0.6 & & 1.6 & & 0.6 & \\
\hline
\end{tabular}


Table 2. Cont

\begin{tabular}{|c|c|c|c|c|c|c|c|c|}
\hline \multirow[b]{2}{*}{$\mathrm{RI}_{\text {calc }}$} & \multirow[b]{2}{*}{$\mathbf{R I}_{\mathrm{db}}$} & \multirow[b]{2}{*}{ Compound } & \multicolumn{6}{|c|}{ Aerial Parts Essential Oil } \\
\hline & & & $\# 1, \%$ & ED, (+):(-) & $\# 2, \%$ & ED, $(+):(-)$ & $\# 3, \%$ & ED, (+):(-) \\
\hline 1558 & 1565 & Eugenyl acetate & $\operatorname{tr}$ & & $\operatorname{tr}$ & & $\operatorname{tr}$ & \\
\hline 1559 & 1557 & Germacrene B & $\operatorname{tr}$ & & - & & - & \\
\hline 1580 & 1577 & Caryophyllene oxide & $\operatorname{tr}$ & & $\operatorname{tr}$ & & 0.1 & \\
\hline 1638 & 1639 & cis-Guaia-3,9-dien-11-ol & 0.1 & & 0.1 & & - & \\
\hline \multirow[t]{8}{*}{1651} & 1655 & $\alpha$-Cadinol & $\operatorname{tr}$ & & 0.1 & & $\operatorname{tr}$ & \\
\hline & & Monoterpene hydrocarbons & 22.8 & & 18.3 & & 31.4 & \\
\hline & & Oxygenated monoterpenoids & 72.2 & & 75.7 & & 61.2 & \\
\hline & & Sesquiterpene hydrocarbons & 1.7 & & 2.2 & & 1.7 & \\
\hline & & Oxygenated sesquiterpenoids & 0.1 & & 0.1 & & 0.1 & \\
\hline & & Benzenoid aromatics & 0.1 & & 0.1 & & 0.1 & \\
\hline & & Others & 3.1 & & 3.6 & & 4.3 & \\
\hline & & Total identified & 100.0 & & 100.0 & & 98.7 & \\
\hline
\end{tabular}

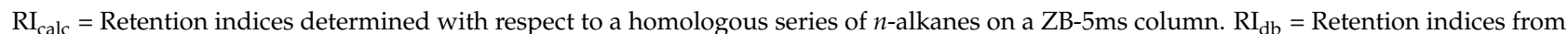
the databases [7-10]. \#1 = Plant sample \#1. \#2 = Plant sample \#2. \#3 = Plant sample \#3. - = Not observed. ED = Enantiomeric distribution (dextrorotatory enantiomer: levorotatory enantiomer). $\operatorname{tr}=$ Trace $(<0.05 \%)$.

As was observed in M. citriodora essential oils, in M. fistulosa essential oils, the (+)enantiomer was the major for $\alpha$-thujene, $\alpha$-pinene, $\beta$-pinene, $\alpha$-phellandrene, $\delta$-3-carene, $\alpha$-terpinene, cis-sabinene hydrate, trans-sabinene hydrate, $\alpha$-terpineol, $\alpha$-copaene, $(E)$ $\beta$-caryophyllene, and germacrene $D$, while the (-)-enantiomer was predominant for $\beta$ phellandrene and borneol. (-)-Limonene (97.4-99.5\%) and (-)-linalool (62.1-62.5\%) dominated in all three $M$. fistulosa samples. (+)-Camphene (100\%), (+)-sabinene (58.4-59.0\%), and (+)-terpinen-4-ol (63.2-63.3\%) were also dominant.

\subsection{Monarda Punctata}

Hydrodistillation of two samples of wild-growing $M$. punctata aerial parts gave bright orange essential oils in $0.781 \%$ and $0.658 \%$ yield. The most abundant components in the essential oils were thymol $\left(\mathrm{RI}_{\mathrm{db}}=1289,61.8 \%\right.$ and $\left.47.9 \%\right)$, $p$-cymene $\left(\mathrm{RI}_{\mathrm{db}}=1024,15.3 \%\right.$ and 19.8\%), $\gamma$-terpinene $\left(\mathrm{RI}_{\mathrm{db}}=1057,2.7 \%\right.$ and $\left.9.7 \%\right)$, and carvacrol $\left(\mathrm{RI}_{\mathrm{db}}=1296,4.5 \%\right.$ and $4.1 \%$ ) (see Table 3).

The enantiomeric distributions of terpenoids in M. punctata essential oils were analogous to those observed for M. citriodora and M. fistulosa oils with the exception of limonene, which was virtually racemic in sample \#1, but 100\% (-)-limonene in sample \#2.

Table 3. Chemical composition of Monarda punctata essential oils growing wild in south Alabama.

\begin{tabular}{|c|c|c|c|c|c|c|}
\hline \multirow[b]{2}{*}{$\mathrm{RI}_{\text {calc }}$} & \multirow[b]{2}{*}{$\mathbf{R I}_{\mathrm{db}}$} & \multirow[b]{2}{*}{ Compound } & \multicolumn{4}{|c|}{ Aerial Parts Essential Oil } \\
\hline & & & $\# 1, \%$ & ED, (+):(-) & $\# 2, \%$ & ED, (+):(-) \\
\hline 923 & 925 & $\alpha$-Thujene & 0.1 & $100: 0$ & 0.7 & $68.5: 31.5$ \\
\hline 930 & 932 & $\alpha$-Pinene & $\operatorname{tr}$ & & 0.2 & $83.8: 16.2$ \\
\hline 945 & 950 & Camphene & - & & 0.1 & 100:0 \\
\hline 957 & 959 & Benzaldehyde & - & & $\operatorname{tr}$ & \\
\hline 970 & 971 & Sabinene & $\operatorname{tr}$ & & $\operatorname{tr}$ & \\
\hline 974 & 978 & $\beta$-Pinene & $\operatorname{tr}$ & & 0.1 & $62.7: 37.3$ \\
\hline 975 & 974 & 1-Octen-3-ol & 1.8 & & 1.8 & \\
\hline 980 & 983 & 3-Octanone & - & & $\operatorname{tr}$ & \\
\hline 986 & 989 & Myrcene & 0.3 & & 1.1 & \\
\hline 995 & 996 & 3-Octanol & 0.1 & & 0.1 & \\
\hline 1001 & 1004 & $p$-Mentha-1(7),8-diene & - & & $\operatorname{tr}$ & \\
\hline 1004 & 1006 & $\alpha$-Phellandrene & 0.1 & 100:0 & 0.2 & $94.6: 5.4$ \\
\hline 1006 & 1008 & 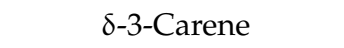 & $\operatorname{tr}$ & & 0.1 & 100:0 \\
\hline 1014 & 1017 & $\alpha$-Terpinene & 1.3 & $100: 0$ & 3.0 & $100: 0$ \\
\hline 1016 & 1022 & m-Cymene & - & & $\operatorname{tr}$ & \\
\hline 1024 & 1024 & $p$-Cymene & 15.3 & & 19.8 & \\
\hline 1025 & 1026 & 2-Acetyl-3-methylfuran & - & & $\operatorname{tr}$ & \\
\hline
\end{tabular}


Table 3. Cont.

\begin{tabular}{|c|c|c|c|c|c|c|}
\hline \multirow[b]{2}{*}{$\mathbf{R I}_{\text {calc }}$} & \multirow[b]{2}{*}{$\mathbf{R} \mathbf{I}_{\mathrm{db}}$} & \multirow[b]{2}{*}{ Compound } & \multicolumn{4}{|c|}{ Aerial Parts Essential Oil } \\
\hline & & & $\# 1, \%$ & $\mathrm{ED},(+):(-)$ & $\# 2, \%$ & $\mathrm{ED},(+):(-)$ \\
\hline 1026 & 1030 & Limonene & 0.4 & $50.2: 49.8$ & 0.5 & $0: 100$ \\
\hline 1027 & 1029 & $\beta$-Phellandrene & 0.2 & & 0.2 & $0: 100$ \\
\hline 1028 & 1030 & 1,8-Cineole & 0.4 & & 0.1 & \\
\hline 1039 & 1043 & Phenylacetaldehyde & - & & $\operatorname{tr}$ & \\
\hline 1055 & 1057 & $\gamma$-Terpinene & 2.0 & & 9.7 & \\
\hline 1066 & 1069 & cis-Sabinene hydrate & 0.6 & 100:0 & 0.7 & 97.9:2.1 \\
\hline 1076 & 1079 & 1-Nonen-3-ol & - & & $\operatorname{tr}$ & \\
\hline 1082 & 1086 & Terpinolene & 0.1 & & 0.1 & \\
\hline 1087 & 1091 & $p$-Cymenene & 0.1 & & 0.1 & \\
\hline 1095 & 1099 & Linalool & - & & $\operatorname{tr}$ & 70.1:29.9 \\
\hline 1098 & 1101 & trans-Sabinene hydrate & 0.2 & $100: 0$ & 0.1 & $83.2: 16.8$ \\
\hline 1100 & 1104 & Nonanal & - & & 0.1 & \\
\hline 1104 & 1107 & 1-Octen-3-yl acetate & 0.2 & & 0.4 & \\
\hline 1145 & 1145 & trans-Verbenol & - & & $\operatorname{tr}$ & \\
\hline 1161 & 1158 & Menthone & 0.3 & & - & \\
\hline 1168 & 1170 & Borneol & 0.1 & $0: 100$ & $\operatorname{tr}$ & $0: 100$ \\
\hline 1177 & 1180 & Terpinen-4-ol & 0.8 & $58.7: 41.3$ & 0.6 & 66.1:33.9 \\
\hline 1182 & 1183 & $m$-Cymen-8-ol & - & & 0.1 & \\
\hline 1184 & 1186 & $p$-Cymen-8-ol & 0.5 & & 0.5 & \\
\hline 1191 & 1197 & Methyl chavicol (= Estragole) & 0.8 & & - & \\
\hline 1193 & 1195 & $\alpha$-Terpineol & - & & 0.1 & 100:0 \\
\hline 1202 & 1206 & Decanal & - & & 0.1 & \\
\hline 1224 & 1224 & Thymol methyl ether & - & & $\operatorname{tr}$ & \\
\hline 1235 & 1238 & Carvacrol methyl ether & 1.1 & & 1.0 & \\
\hline 1239 & 1242 & Cumin aldehyde & - & & 0.1 & \\
\hline 1247 & 1250 & Thymoquinone & 2.0 & & 0.2 & \\
\hline 1289 & 1289 & Thymol & 61.8 & & 47.9 & \\
\hline 1293 & 1291 & $p$-Cymen-7-ol & - & & 0.2 & \\
\hline 1296 & 1296 & Carvacrol & 4.5 & & 4.1 & \\
\hline 1306 & 1309 & 4-Vinylguaicol & - & & $\operatorname{tr}$ & \\
\hline 1347 & 1356 & Eugenol & 0.7 & & 0.3 & \\
\hline 1370 & 1375 & $\alpha$-Copaene & - & & $\operatorname{tr}$ & \\
\hline 1378 & 1382 & $\beta$-Bourbonene & - & & $\operatorname{tr}$ & \\
\hline 1384 & 1390 & trans- $\beta$-Elemene & - & & $\operatorname{tr}$ & \\
\hline 1415 & 1417 & $(E)-\beta$-Caryophyllene & 1.6 & 100:0 & 1.2 & $100: 0$ \\
\hline 1424 & 1430 & $\beta$-Copaene & - & & $\operatorname{tr}$ & \\
\hline 1427 & 1430 & trans- $\alpha$-Bergamotene & 1.2 & & 0.7 & \\
\hline 1449 & 1453 & $\alpha$-Humulene & - & & $\operatorname{tr}$ & \\
\hline 1469 & 1475 & $\gamma$-Muurolene & - & & 0.1 & \\
\hline 1476 & 1480 & Germacrene D & 0.7 & 100:0 & 0.4 & 100:0 \\
\hline 1479 & 1483 & trans- $\beta$-Bergamotene & 0.2 & & 0.1 & \\
\hline 1480 & 1485 & $\gamma$-Thujaplicin & - & & 0.1 & \\
\hline 1482 & 1489 & $\beta$-Selinene & - & & $\operatorname{tr}$ & \\
\hline 1489 & 1492 & $\alpha$-Selinene & - & & $\operatorname{tr}$ & \\
\hline 1492 & 1497 & $\alpha$-Muurolene & - & & $\operatorname{tr}$ & \\
\hline 1506 & 1512 & $\gamma$-Cadinene & - & & $\operatorname{tr}$ & \\
\hline 1512 & 1518 & $\delta$-Cadinene & - & & 0.1 & \\
\hline 1517 & 1523 & $\beta$-Sesquiphellandrene & - & & 0.1 & \\
\hline 1546 & 1549 & Thymohydroquinone & 0.3 & & 2.5 & \\
\hline 1576 & 1577 & Caryophyllene oxide & 0.2 & & 0.2 & \\
\hline 1633 & 1639 & cis-Guaia-3,9-dien-11-ol & 0.2 & & 0.1 & \\
\hline 1648 & 1655 & $\alpha$-Cadinol & - & & $\operatorname{tr}$ & \\
\hline \multirow[t]{5}{*}{1834} & 1841 & Phytone & - & & $\operatorname{tr}$ & \\
\hline & & Monoterpene hydrocarbons & 19.9 & & 35.7 & \\
\hline & & Oxygenated monoterpenoids & 72.5 & & 58.3 & \\
\hline & & Sesquiterpene hydrocarbons & 3.7 & & 2.7 & \\
\hline & & Oxygenated sesquiterpenoids & 0.4 & & 0.4 & \\
\hline
\end{tabular}


Table 3. Cont

\begin{tabular}{|c|c|c|c|c|c|c|}
\hline \multirow[b]{2}{*}{$\mathbf{R I}_{\text {calc }}$} & \multirow[b]{2}{*}{$\mathbf{R I}_{\mathrm{db}}$} & \multirow[b]{2}{*}{ Compound } & \multicolumn{4}{|c|}{ Aerial Parts Essential Oil } \\
\hline & & & $\# 1, \%$ & ED, (+):(-) & $\# 2, \%$ & ED, (+):(-) \\
\hline & & Benzenoid aromatics & 1.5 & & 0.3 & \\
\hline & & Others & 2.1 & & 2.4 & \\
\hline & & Total identified & 100.0 & & 99.8 & \\
\hline
\end{tabular}

$\mathrm{RI}_{\text {calc }}=$ Retention indices determined with respect to a homologous series of $n$-alkanes on a ZB-5ms column. $\mathrm{RI}_{\mathrm{db}}=\mathrm{Retention} \mathrm{indices}$ from the databases [7-10]. \#1 = Plant sample \#1. \#2 = Plant sample \#2. - = Not observed. ED = Enantiomeric distribution (dextrorotatory enantiomer: levorotatory enantiomer). $\operatorname{tr}=$ Trace $(<0.05 \%)$.

\section{Discussion}

Monarda citriodora and $M$. fistulosa have been introduced throughout temperate regions of the world as popular herbal medicines as well as ornamentals [4-6]. The volatile phytochemistry has shown wide variation depending on geographical location (Table 4). The essential oils of $M$. citriodora in the present study were rich in both thymol and carvacrol, whereas essential oils from Europe and Asia were dominated by thymol with much lower concentrations of carvacrol. Monarda fistulosa, in particular, showed wide variation with at least three different chemotypes (carvacrol-rich, thymol-rich, and geraniol-rich, see Table 4). The essential oils of $M$. fistulosa (samples \#1 and \#2) in this study fit into the thymol-rich chemotype. Interestingly, there was a high concentration of thymoquinone in $M$. fistulosa sample \#3, with concomitant lower concentrations of thymol and carvacrol. Thymol was reported as the major component of $M$. punctata in two old reports [11,12]. Consistent with these reports, a floral essential oil of $M$. punctata from China was rich in thymol (75.2\%), which is in agreement with the aerial parts essential oils from Alabama.

Table 4. Major essential oil components of Monarda species from geographical locations around the world.

\begin{tabular}{|c|c|c|c|c|}
\hline Monarda spp. & Plant Tissue & Collection Site & Composition (Major Components) & Ref. \\
\hline M. citriodora & Aerial parts & $\begin{array}{l}\text { Jammu, India } \\
\text { (cultivated) }\end{array}$ & Thymol (82.3\%), carvacrol (4.8\%) & [13] \\
\hline M. citriodora & Aerial parts & $\begin{array}{l}\text { Imola (BO) Italy } \\
\quad \text { (cultivated) }\end{array}$ & $\begin{array}{c}\text { Thymol }(19.6 \%), p \text {-cymene }(15.6 \%), \\
\gamma \text {-terpinene }(13.5 \%), \text { carvacrol }(9.3 \%) \\
\alpha \text {-terpinene }(9.2 \%), \text { myrcene }(5.7 \%)\end{array}$ & [14] \\
\hline M. citriodora & Not reported & Commercial (India) & $\begin{array}{c}(E)-\beta \text {-Caryophyllene }(19.2 \%) \text {, citral }{ }^{\text {a }} \\
(13.3 \%), \text { limonene }(11.8 \%), \text { cis-verbenol } \\
(11.4 \%) \text {, geraniol }(7.6 \%) \\
\text { citronellal }(5.6 \%)\end{array}$ & [15] \\
\hline $\begin{array}{l}\text { M. citriodora var. } \\
\text { citriodora }\end{array}$ & Leaves & $\begin{array}{l}\text { Liverpool, UK } \\
\text { (cultivated) }\end{array}$ & $\begin{array}{c}\text { Thymol }(50.7 \%), p \text {-cymene }(22.8 \%) \\
\text { carvacrol }(3.6 \%)\end{array}$ & [16] \\
\hline $\begin{array}{l}\text { M. citriodora var. } \\
\text { citriodora }\end{array}$ & Flowers & $\begin{array}{l}\text { Liverpool, UK } \\
\text { (cultivated) }\end{array}$ & $\begin{array}{c}\text { Thymol }(61.8 \%), \gamma \text {-terpinene }(13.3 \%), \\
p \text {-cymene }(4.2 \%), \text { carvacrol }(3.8 \%)\end{array}$ & [16] \\
\hline $\begin{array}{l}\text { M. citriodora var. } \\
\text { citriodora }\end{array}$ & Aerial parts & $\begin{array}{l}\text { Liverpool, UK } \\
\text { (cultivated) }\end{array}$ & $\begin{array}{l}\text { Thymol }(56.9 \%), p \text {-cymene }(13.0 \%) \text {, } \\
\alpha \text {-terpinene }(10.0 \%) \text {, carvacrol }(4.3 \%)\end{array}$ & [17] \\
\hline $\begin{array}{l}\text { M. citriodora var. } \\
\text { citriodora }\end{array}$ & Aerial parts & $\begin{array}{l}\text { Commercial } \\
\text { (unknown) }\end{array}$ & $\begin{array}{c}\text { Thymol }(70.6 \%), p \text {-cymene }(10.6 \%) \\
\text { carvacrol }(6.1 \%)\end{array}$ & [18] \\
\hline M. fistulosa & Aerial parts & $\begin{array}{l}\text { Krasnodarsk Krai, } \\
\text { Russia (introduced, } \\
\text { wild) }\end{array}$ & $\begin{array}{c}\text { p-Cymene }(32.5 \%) \text {, carvacrol }(23.9 \%) \\
\text { thymol }(12.6 \%) \text {, carvacrol methyl ether } \\
(5.5 \%) \text {, unidentified aliphatic } \\
\text { aldehyde }(6.3 \%)\end{array}$ & [19] \\
\hline M. fistulosa & Aerial parts & $\begin{array}{l}\text { Casola Valsenio, Italy } \\
\text { (cultivated) }\end{array}$ & $\begin{array}{l}\text { Thymol (26.5\%), } \beta \text {-phellandrene } \\
(17.0 \%), \alpha \text {-phellandrene }(13.7 \%), \\
\text { p-cymene }(13.5 \%), \text { myrcene }(8.1 \%)\end{array}$ & [20] \\
\hline M. fistulosa & Aerial parts & $\begin{array}{c}\text { Saint-Jean-sur- } \\
\text { Richelieu, QC, Canada } \\
\text { (cultivated) }\end{array}$ & $\begin{array}{c}\text { Geraniol }(61.8 \%) \text {, geranyl formate } \\
(16.6 \%) \text {, geranial }(10.6 \%) \text {, neral }(6.6 \%)\end{array}$ & [21] \\
\hline M. fistulosa & Aerial parts & $\begin{array}{l}\text { Poplarville, MS, USA } \\
\text { (cultivated) }\end{array}$ & $\begin{array}{c}\text { Carvacrol }(39.1 \%), p \text {-cymene }(35.4 \%) \\
(-)-1 \text {-octen-3-ol }\end{array}$ & [22] \\
\hline
\end{tabular}


Table 4. Cont.

\begin{tabular}{|c|c|c|c|c|}
\hline Monarda spp. & Plant Tissue & Collection Site & Composition (Major Components) & Ref. \\
\hline M. fistulosa & Aerial parts & $\begin{array}{l}\text { Imola (BO) Italy } \\
\quad \text { (cultivated) }\end{array}$ & $\begin{array}{l}\text { Thymol (31.6\%), } \beta \text {-phellandrene } \\
(18.1 \%), \alpha \text {-phellandrene }(14.2 \%), \\
\text { p-cymene }(13.1 \%), \text { myrcene }(8.8 \%)\end{array}$ & [23] \\
\hline M. fistulosa & Aerial parts & $\begin{array}{l}\text { Imola (BO) Italy } \\
\text { (cultivated) }\end{array}$ & $\begin{array}{l}\text { Thymol }(28.4 \%), \beta \text {-phellandrene } \\
(16.9 \%), \alpha \text {-phellandrene }(13.7 \%), \\
\text { p-cymene }(13.3 \%), \text { myrcene }(8.7 \%)\end{array}$ & [24] \\
\hline M. fistulosa & Aerial parts & $\begin{array}{l}\text { Imola (BO) Italy } \\
\text { (cultivated) }\end{array}$ & $\begin{array}{l}\text { Thymol (33.4\%), } \beta \text {-phellandrene } \\
(18.0 \%), \alpha \text {-phellandrene }(14.0 \%), \\
\text { p-cymene }(13.2 \%), \text { myrcene }(8.6 \%)\end{array}$ & [24] \\
\hline M. fistulosa & Aerial parts & $\begin{array}{l}\text { Ravenna, Italy } \\
\text { (cultivated) }\end{array}$ & $\begin{array}{c}\gamma \text {-Terpinene }(25.2 \%) \text {, carvacrol }(24.3 \%), \\
p \text {-cymene }(11.0 \% \text {; reported as } o \text {-cymene }) \\
\text { thymol }(8.4 \%), \alpha \text {-terpinene }(5.0 \%) \\
\text { thymol methyl ether }(4.7 \%)\end{array}$ & [25] \\
\hline M. fistulosa & Aerial parts & $\begin{array}{l}\text { Chişinău, Republic of } \\
\text { Moldova (cultivated) }\end{array}$ & $\begin{array}{l}\text { Carvacrol }(54.8 \%), p \text {-cymene }(23.2 \%) \\
\text { carvacrol methyl ether }(5.9 \%)\end{array}$ & [26] \\
\hline M. fistulosa & Flowers & $\begin{array}{l}\text { Gallatin Valley, MT, } \\
\text { USA (wild) }\end{array}$ & $\begin{array}{c}\text { Carvacrol }(45.7 \%), p \text {-cymene }(25.6 \%) \\
\gamma \text {-terpinen }(6.8 \%) \text {, thymol }(3.1 \%)\end{array}$ & [27] \\
\hline M. fistulosa & Leaves & $\begin{array}{l}\text { Gallatin Valley, MT, } \\
\text { USA (wild) }\end{array}$ & $\begin{array}{c}\text { Carvacrol }(71.5 \%), p \text {-cymene }(13.1 \%) \\
\gamma \text {-terpinen }(2.5 \%), \text { thymol }(3.3 \%)\end{array}$ & [27] \\
\hline M. fistulosa & Aerial parts & $\begin{array}{l}\text { Moscow, Russia } \\
\text { (cultivated) }\end{array}$ & $\begin{array}{c}\alpha \text {-Terpineol }(37.7 \%), 1 \text {-octen-3-ol } \\
(10.5 \%) \text {, geraniol }(10.4 \%) \text {, thymol }(9.3 \%), \\
\text { p-cymene }(4.9 \%)\end{array}$ & [28] \\
\hline M. fistulosa cv. Fortuna & Aerial parts & $\begin{array}{l}\text { Kherson, Ukraine } \\
\text { (cultivated) }\end{array}$ & $\begin{array}{c}\text { Thymol }(77.3 \%) \text {, carvacrol methyl ether } \\
(4.9 \%) \text {, carvacrol }(3.8 \%)\end{array}$ & [6] \\
\hline M. fistulosa cv. Premiera & Aerial parts & $\begin{array}{l}\text { Kherson, Ukraine } \\
\text { (cultivated) }\end{array}$ & $\begin{array}{c}\text { Thymol }(78.3 \%) \text {, carvacrol methyl ether } \\
(4.8 \%) \text {, carvacrol }(3.6 \%)\end{array}$ & [6] \\
\hline $\begin{array}{l}\text { M. fistulosa var. } \\
\text { menthifolia }\end{array}$ & Aerial parts & $\begin{array}{l}\text { Morden, Manitoba, } \\
\text { Canada (cultivated) }\end{array}$ & Geraniol $(86.8 \%)$ & [29] \\
\hline M. punctata & Flowers & $\begin{array}{l}\text { Xi'an, China } \\
\text { (cultivated?) }\end{array}$ & $\begin{array}{l}\text { Thymol }(75.2 \%), p \text {-cymene }(6.7 \%) \text {, } \\
\text { limonene }(5.4 \%) \text {, carvacrol }(3.5 \%)\end{array}$ & [30] \\
\hline
\end{tabular}

a Isomer not indicated.

The high concentrations of thymol, carvacrol, and $p$-cymene are consistent with the traditional uses of Monarda spp. to treat skin infections, wounds, fevers, and respiratory problems. Thymol [31], carvacrol [32], and $p$-cymene [33] have demonstrated antibacterial and antifungal activities [34,35], as well as wound-healing activity [36]. Thymol [37] and carvacrol [38], in addition to thymoquinone [39], have shown antitussive effects. Thymoquinone has also shown wound-healing properties [40]. Furthermore, both thymol [41] and carvacrol [32] have shown analgesic and anti-inflammatory activities [42].

As far as we are aware, this work presents the first chiral analysis of terpenoid constituents of Monarda species. Several investigations on the enantiomeric distributions in other members of the Lamiaceae have been reported in the literature, however. There seems to be much variation in the enantiomeric distribution of monoterpenoids across the family. Consistent with what was observed in Monarda essential oils, $(+)-\alpha$-pinene was the major enantiomer found in Coridothymus capitatus [43], Rosmarinus officinalis [44], Lepechinia heteromorpha [45], Ocimum canum, and Ocimum kilimandscharicum [46]. Likewise, (+)- $\beta$-pinene predominates over (-)- $\beta$-pinene in C. capitatus [43] as well as the Monarda essential oils. On the other hand, (-)- $\beta$-pinene dominates in R. officinalis [44] and Lepechinia mutica [47]. The essential oils of peppermint (Mentha $\times$ piperita) and spearmint (Mentha spicata) have shown nearly racemic mixtures of $\alpha$ - and $\beta$-pinenes [48]. (+)- $\alpha-$ Phellandrene and (-)- $\beta$-phellandrene were the dominant enantiomers in the Monarda essential oils. In marked contrast, however, $(-)-\alpha$-phellandrene and $(+)-\beta$-phellandrene predominated in L. mutica essential oil [47]. (-)-Limonene predominates in M. fistulosa essential oil, peppermint (M. piperita) and spearmint (M. spicata) essential oils [48] whereas (+)-limonene is the major enantiomer in C. capitatus [43], O. canum, and O. kilimandscharicum [46], and a nearly 
racemic mixture was found in rosemary (R. officinalis) essential oil [44]. (+)-Linalool was the predominant enantiomer in C. capitatus [43], Salvia schimperi [49], Pycnanthemum incanum [50], O. canum, and O. kilimandscharicum [46], whereas (-)-linalool was the major stereoisomer in Lavandula angustifolia [51] and R. officinalis [44].

\section{Materials and Methods}

\subsection{Plant Material}

Monarda citriodora was cultivated in Kirkland Gardens, Newville, AL, USA $\left(31^{\circ} 26^{\prime} 27^{\prime \prime}\right.$ N, $85^{\circ} 21^{\prime} 31^{\prime \prime} \mathrm{W}$ ) from seeds (Outsidepride Seed Source, Independence, OR, USA). The cultivated Monarda spp. were grown in loamy clayey-sand and fertilized with chicken manure, kelp meal, and bone meal at planting in full sun. The aerial parts of $M$. citriodora were collected from separate plants on separate occasions (plant \#1, collected on 20 June 2020; plant \#2 collected on 1 August 2020). The roots of $M$. citriodora were obtained from plant \#2.

Monarda fistulosa was cultivated in Kirkland Gardens, Newville, AL, USA ( $31^{\circ} 26^{\prime} 27^{\prime \prime}$ N, $85^{\circ} 21^{\prime} 31^{\prime \prime} \mathrm{W}$ ) from seedlings (Home Depot, Dothan, AL, USA) as above. The aerial parts of three different plant samples were collected on 25 June 2020.

Monarda punctata was collected from wild-growing plants near Newville, AL, USA $\left(31^{\circ} 27^{\prime} 23^{\prime \prime} \mathrm{N}, 85^{\circ} 22^{\prime} 17^{\prime \prime} \mathrm{W}\right)$; the edge of a planted pine forest, disturbed grassland, full/partial sun, sandy-clay soil that had been intentionally burned (prescribed burn) 1.5 years before collection. The aerial parts of two different plants were collected on 1 June 2020.

Plants were identified by S.K. Lawson and a voucher specimen of each plant was deposited in the University of Alabama in Huntsville Herbarium (HALA); voucher numbers for M. citriodora (SKL61820), M. fistulosa (SKL72020), and M. punctata (SKL9620). The Monarda plant materials were allowed to dry in the shade for several days, the airdried plant materials were pulverized and subjected to hydrodistillation using a LikensNickerson apparatus with continuous extraction with dichloromethane (Table 5).

Table 5. Hydrodistillation details of Monarda species collected or cultivated in south Alabama.

\begin{tabular}{ccc}
\hline Monarda spp. & Mass Plant Material & Yield Essential Oil (EO) \\
\hline Monarda citriodora \#1 & $25.57 \mathrm{~g}$ dried aerial parts & $406.2 \mathrm{mg}$ orange EO \\
Monarda citriodora \#2 & $37.81 \mathrm{~g}$ dried aerial parts & $675.6 \mathrm{mg}$ orange EO \\
Monarda citriodora \#2 & $17.47 \mathrm{~g}$ dried roots & $153.6 \mathrm{mg}$ yellow EO \\
Monarda fistulosa \#1 & $9.60 \mathrm{~g}$ dried aerial parts & $364.0 \mathrm{mg}$ bright orange EO \\
Monarda fistulosa \#2 & $7.58 \mathrm{~g}$ dried aerial parts & $366.2 \mathrm{mg}$ bright orange EO \\
Monarda fistulosa \#3 & $8.98 \mathrm{~g}$ dried aerial parts & $238.9 \mathrm{mg}$ bright orange EO \\
Monarda punctata \#1 & $39.09 \mathrm{~g}$ dried aerial parts & $305.6 \mathrm{mg}$ bright orange EO \\
Monarda punctata \#2 & $62.62 \mathrm{~g}$ dried aerial parts & $411.9 \mathrm{mg}$ bright orange EO \\
\hline
\end{tabular}

\subsection{Gas Chromatographic Analysis}

The essential oils were analyzed by gas chromatography-mass spectrometry (GCMS), gas chromatography with flame ionization detection (GC-FID), and chiral GC-MS as previously reported [52].

\subsubsection{Gas Chromatography-Mass Spectrometry}

Shimadzu GCMS-QP2010 Ultra, ZB-5ms GC column, GC oven temperature $50{ }^{\circ} \mathrm{C}-$ $260{ }^{\circ} \mathrm{C}\left(2{ }^{\circ} \mathrm{C} / \mathrm{min}\right), 1-\mu \mathrm{L}$ injection of $5 \%$ solution of $\mathrm{EO}$ in dichloromethane (split mode, 30:1). Retention indices (RIs) were determined with reference to a homologous series of $n$-alkanes. Compounds identified by comparison of the MS fragmentation and retention indices with those in the databases [7-10].

\subsubsection{Gas Chromatography-Flame Ionization Detection}

Shimadzu GC 2010, FID detector, ZB-5 GC column, GC oven temperature $50{ }^{\circ} \mathrm{C}-$ $260{ }^{\circ} \mathrm{C}\left(2.0^{\circ} \mathrm{C} / \mathrm{min}\right)$. The percent compositions were determined from raw peak areas without standardization. 


\subsubsection{Chiral Gas Chromatography-Mass Spectrometry}

Shimadzu GCMS-QP2010S, Restek B-Dex 325 column, GC oven temperature $50{ }^{\circ} \mathrm{C}$ $120{ }^{\circ} \mathrm{C}\left(1.5^{\circ} \mathrm{C} / \mathrm{min}\right)$ then $120^{\circ} \mathrm{C}-200^{\circ} \mathrm{C}\left(2.0^{\circ} \mathrm{C} / \mathrm{min}\right), 0.1 \mu \mathrm{L}$ injection of $5 \%$ solution of $\mathrm{EO}$ in dichloromethane (split mode, 45:1). The enantiomeric distributions were determined by comparison of retention times with authentic samples obtained from Sigma-Aldrich (Milwaukee, WI, USA). Relative enantiomer percentages were calculated from peak areas.

\section{Conclusions}

This study presents, for the first time, analyses of the essential oils of three species of Monarda growing in south Alabama. In addition, the enantiomeric distribution of terpenoids was also carried out. This work illustrates the wide variation in essential oil compositions based on geographical location as well as variations in enantiomeric distribution. It would be interesting to compare enantiomeric distributions for Monarda essential oils from other geographical locations and for other Monarda species. Nevertheless, the phenolic monoterpenoids thymol and/or carvacrol were found to dominate the compositions of M. citriodora, M. fistulosa, and M. punctata and support the traditional medicinal uses of these plants.

Author Contributions: Conceptualization, S.K.L. and W.N.S.; methodology, S.K.L., P.S., and W.N.S.; software, P.S.; validation, W.N.S., formal analysis, P.S. and W.N.S.; investigation, S.K.L., P.S., and W.N.S.; data curation, W.N.S.; writing—original draft preparation, W.N.S.; writing—review and editing, S.K.L., P.S., and W.N.S. All authors have read and agreed to the published version of the manuscript.

Funding: This research received no external funding.

Institutional Review Board Statement: Not applicable.

Informed Consent Statement: Not applicable.

Data Availability Statement: All data are contained within the article.

Acknowledgments: P.S. and W.N.S. participated in this work as part of the activities of the Aromatic Plant Research Center (APRC, https:/ / aromaticplant.org/, accessed on 9 February 2021).

Conflicts of Interest: The authors declare no conflict of interest.

\section{References}

1. Royal Botanic Gardens, K. The Plant List. Available online: http:/ /www.theplantlist.org/tpl1.1/search?q=Monarda (accessed on 1 February 2021).

2. Kartesz, J.T. BONAP's North American Plant Atlas. Available online: http://bonap.net/Napa/TaxonMaps/Genus/County/ Monarda (accessed on 1 February 2021).

3. Moerman, D.E. Native American Ethnobotany; Timber Press, Inc.: Portland, OR, USA, 1998.

4. Davidson, C.G. Monarda, Bee-balm. In Flower Breeding and Genetics; Anderson, N.O., Ed.; Springer: Dordrecht, The Netherlands, 2007; pp. 756-779. ISBN 978-1-4020-4427-4.

5. Ciuruşniuc, A.-M.; Robu, T. Study of the behaviour of cultivated species of the genus Monarda L. in Vaslui County, to introduce them in cultivation as medicinal, aromatic and decorative plants. Lucr. Stiintifice Ser. Agron. 2012, 55, 309-312.

6. Dudchenko, V.V.; Svydenko, L.V.; Markovska, O.Y.; Sydiakina, O.V. Morphobiological and biochemical characteristics of Monarda L. varieties under conditions of the southern steppe of Ukraine. J. Ecol. Eng. 2020, 21, 99-107.

7. Adams, R.P. Identification of Essential Oil Components by Gas Chromatography/Mass Spectrometry, 4th ed.; Allured Publishing: Carol Stream, IL, USA, 2007; ISBN 978-1-932633-21-4.

8. Mondello, L. FFNSC 3; Shimadzu Scientific Instruments: Columbia, MD, USA, 2016.

9. NIST. NIST17; National Institute of Standards and Technology: Gaithersburg, MD, USA, 2017.

10. Satyal, P. Development of GC-MS Database of Essential Oil Components by the Analysis of Natural Essential Oils and Synthetic Compounds and Discovery of Biologically Active Novel Chemotypes in Essential Oils. Ph.D. Thesis, University of Alabama in Huntsville, Huntsville, AL, USA, 2015.

11. Schroeter, H.J. Analysis of the volatile oil of Monarda punctata, Linne. Am. J. Pharm. 1888, 1888, 113.

12. Schumann, W.R.; Kremers, E. On the chemical composition of the oil from Monarda punctata, L. Am. J. Pharm. 1896, 1896, 469. 
13. Pathania, A.S.; Guru, S.K.; Verma, M.K.; Sharma, C.; Abdullah, S.T.; Malik, F.; Chandra, S.; Katoch, M.; Bhushan, S. Disruption of the PI3K/AKT/mTOR signaling cascade and induction of apoptosis in HL-60 cells by an essential oil from Monarda citriodora. Food Chem. Toxicol. 2013, 62, 246-254. [CrossRef]

14. Di Vito, M.; Bellardi, M.G.; Mondello, F.; Modesto, M.; Michelozzi, M.; Bugli, F.; Sanguinetti, M.; Sclocchi, M.C.; Sebastiani, M.L.; Biffi, S.; et al. Monarda citriodora hydrolate vs. essential oil comparison in several anti-microbial applications. Ind. Crops Prod. 2019, 128, 206-212. [CrossRef]

15. Deepika; Singh, A.; Chaudhari, A.K.; Das, S.; Dubey, N.K. Nanoencapsulated Monarda citriodora Cerv. ex Lag. essential oil as potential antifungal and antiaflatoxigenic agent against deterioration of stored functional foods. J. Food Sci. Technol. 2020, 57, 2863-2876. [CrossRef] [PubMed]

16. Collins, J.E.; Bishop, C.D.; Deans, S.G.; Svoboda, K.P. Composition of the essential oil from the leaves and flowers of Monarda citriodora var. citriodora grown in the United Kingdom. J. Essent. Oil Res. 1994, 6, 27-29. [CrossRef]

17. Bishop, C.D.; Thornton, I.B. Evaluation of the antifungal activity of the essential oils of Monarda citriodora var. citriodora and Melaleuca alternifolia on post-harvest pathogens. J. Essent. Oil Res. 1997, 9, 77-82. [CrossRef]

18. Dorman, H.J.D.; Deans, S.G. Chemical composition, antimicrobial and in vitro antioxidant properties of Monarda citriodora var. citriodora, Myristica fragrans, Origanum vulgare ssp. hirtum, Pelargonium sp. and Thymus zygis oils. J. Essent. Oil Res. 2004, 16, 145-150. [CrossRef]

19. Zamureenko, V.A.; Klyuev, N.A.; Bocharov, B.V.; Kabanov, V.S.; Zacharov, A.M. An investigation of the component composition of the essential oil of Monarda fistulosa. Chem. Nat. Compd. 1989, 25, 549-551. [CrossRef]

20. Contaldo, N.; Bellardi, M.G.; Cavicchi, L.; Epifano, F.; Genovese, S.; Curini, M.; Bertaccini, A. Phytochemical effects of phytoplasma infections on essential oil of Monarda fistulosa L. Bull. Insectology 2011, 64, S177-S178.

21. Adebayo, O.; Bélanger, A.; Khanizadeh, S. Variable inhibitory activities of essential oils of three Monarda species on the growth of Botrytis cinerea. Can. J. Plant Sci. 2013, 93, 987-995. [CrossRef]

22. Tabanca, N.; Bernier, U.R.; Ali, A.; Wang, M.; Demirci, B.; Blythe, E.K.; Khan, S.I.; Baser, K.H.C.; Khan, I.A. Bioassay-guided investigation of two Monarda essential oils as repellents of yellow fever mosquito Aedes aegypti. J. Agric. Food Chem. 2013, 61, 8573-8580. [CrossRef]

23. Francati, S.; Gualandi, G. Side effects of essential oils of Monarda fistulosa L. and M. didyma L. on the tachinid parasitoid Exorista larvarum (L.): A preliminary study. Tachinid Times 2017, 30, 4-8.

24. Mattarelli, P.; Epifano, F.; Minardi, P.; Di Vito, M.; Modesto, M.; Barbanti, L.; Bellardi, M.G. Chemical composition and antimicrobial activity of essential oils from aerial parts of Monarda didyma and Monarda fistulosa cultivated in Italy. J. Essent. Oil-Bear. Plants 2017, 20, 76-86. [CrossRef]

25. Laquale, S.; Avato, P.; Argentieri, M.P.; Bellardi, M.G.; D'Addabbo, T. Nematotoxic activity of essential oils from Monarda species. J. Pest Sci. 2018, 91, 1115-1125. [CrossRef]

26. Colţun, M.B.; Bogdan, A.A. Aspects of the biology and the cultivation of Monarda fistulosa L. as aromatic species in the Republic Of Moldova. Основні, малопоширені і нетрадиційні види рослин-від вивчення до освоєння(сільськогосподарські і біологічні науки) 2020, 2020, 103-109.

27. Ghosh, M.; Schepetkin, I.A.; Özek, G.; Özek, T.; Khlebnikov, A.I.; Damron, D.S.; Quinn, M.T. Essential oils from Monarda fistulosa: Chemical composition and activation of transient receptor potential A1 (TRPA1) channels. Molecules 2020, 25, 4873. [CrossRef] [PubMed]

28. Malankina, E.L.; Kuzmenko, A.N.; Zaitchik, B.T.; Ruzhitskiy, A.O.; Evgrafov, A.A.; Kozlovskaya, L.N. Content and composition of wild bergamot (Monarda fistulosa L.) essential oil at different phenological phases. Moscow Univ. Chem. Bull. 2020, 75, 391-394. [CrossRef]

29. Mazza, G.; Chubey, B.B.; Kiehn, F. Essential oil of Monarda fistulosa L. var. menthaefolia, a potential source of geraniol. Flavour Fragr. J. 1987, 2, 129-132. [CrossRef]

30. Li, H.; Yang, T.; Li, F.-Y.; Yao, Y.; Sun, Z.-M. Antibacterial activity and mechanism of action of Monarda punctata essential oil and its main components against common bacterial pathogens in respiratory tract. Int. J. Clin. Exp. Pathol. 2014, 7, 7389-7398.

31. Marchese, A.; Orhan, I.E.; Daglia, M.; Barbieri, R.; Di Lorenzo, A.; Nabavi, S.F.; Gortzi, O.; Izadi, M.; Nabavi, S.M. Antibacterial and antifungal activities of thymol: A brief review of the literature. Food Chem. 2016, 210, 402-414. [CrossRef] [PubMed]

32. Sharifi-Rad, M.; Varoni, E.M.; Iriti, M.; Martorell, M.; Setzer, W.N.; del Mar Contreras, M.; Salehi, B.; Soltani-Nejad, A.; Rajabi, S.; Tajbakhsh, M.; et al. Carvacrol and human health: A comprehensive review. Phyther. Res. 2018, 32, 1675-1687. [CrossRef] [PubMed]

33. Marchese, A.; Arciola, C.R.; Barbieri, R.; Silva, A.S.; Nabavi, S.F.; Sokeng, A.J.T.; Izadi, M.; Jafari, N.J.; Suntar, I.; Daglia, M.; et al. Update on monoterpenes as antimicrobial agents: A particular focus on $p$-cymene. Materials 2017, 10, 947. [CrossRef] [PubMed]

34. Memar, M.Y.; Raei, P.; Alizadeh, N.; Aghdam, M.A.; Kafil, H.S. Carvacrol and thymol: Strong antimicrobial agents against resistant isolates. Rev. Med. Microbiol. 2017, 28, 63-68. [CrossRef]

35. Aljaafari, M.N.; AlAli, A.O.; Baqais, L.; Alqubaisy, M.; AlAli, M.; Aidin, M.; Ong-Abdullah, J.; Abushelaibi, A.; Lai, K.-S.; Lim, S.-H.E. An overview of the potential therapeutic applications of essential oils. Molecules 2021, 26, 628. [CrossRef] [PubMed]

36. Costa, M.F.; Durço, A.O.; Rabelo, T.K.; Barreto, R.d.S.S.; Guimarães, A.G. Effects of carvacrol, thymol and essential oils containing such monoterpenes on wound healing: A systematic review. J. Pharm. Pharmacol. 2019, 71, 141-155. [CrossRef] [PubMed] 
37. Gavliakova, S.; Biringerova, Z.; Buday, T.; Brozmanova, M.; Calkovsky, V.; Poliacek, I.; Plevkova, J. Antitussive effects of nasal thymol challenges in healthy volunteers. Respir. Physiol. Neurobiol. 2013, 187, 104-107. [CrossRef]

38. Boskabady, M.H.; Jandaghi, P.; Kiani, S.; Hasanzadeh, L. Antitussive effect of Carum copticum in guinea pigs. J. Ethnopharmacol. 2005, 97, 79-82. [CrossRef]

39. Hosseinzadeh, H.; Eskandari, M.; Ziaee, T. Antitussive effect of thymoquinone, a constituent of Nigella sativa seeds, in Guinea pigs. Pharmacologyonline 2008, 2, 480-484.

40. Selçuk, C.T.; Durgun, M.; Tekin, R.; Yolbas, L.; Bozkurt, M.; Akçay, C.; Alabalk, U.; Basarali, M.K. Evaluation of the effect of thymoquinone treatment on wound healing in a rat burn model. J. Burn Care Res. 2013, 34, 274-281. [CrossRef] [PubMed]

41. Jyoti, D.D.; Singh, D.; Kumar, G.; Karnatak, M.; Chandra, S.; Verma, V.P.; Shankar, R. Thymol chemistry: A medicinal toolbox. Curr. Bioact. Compd. 2018, 15, 454-474. [CrossRef]

42. Fachini-Queiroz, F.C.; Kummer, R.; Estevão-Silva, C.F.; Carvalho, M.D.D.B.; Cunha, J.M.; Grespan, R.; Bersani-Amado, C.A.; Cuman, R.K.N. Effects of thymol and carvacrol, constituents of Thymus vulgaris L. essential oil, on the inflammatory response. Evid. Based Complement. Altern. Med. 2012, 2012, 657026. [CrossRef]

43. Tateo, F.; Mariotti, M.; Bononi, M. Essential oil composition and enantiomeric distribution of some monoterpenoid components of Coridothymus capitatus (L.) Rchb. grown on the island of Kos (Greece). J. Essent. Oil Res. 1998, 10, 241-244. [CrossRef]

44. Satyal, P.; Jones, T.H.; Lopez, E.M.; McFeeters, R.L.; Ali, N.A.A.; Mansi, I.; Al-Kaf, A.G.; Setzer, W.N. Chemotypic characterization and biological activity of Rosmarinus officinalis. Foods 2017, 6, 20. [CrossRef] [PubMed]

45. Gilardoni, G.; Ramírez, J.; Montalván, M.; Quinche, W.; León, J.; Benítez, L.; Morocho, V.; Cumbicus, N.; Bicchi, C. Phytochemistry of three Ecuadorian Lamiaceae: Lepechinia heteromorpha (Briq.) Epling, Lepechinia radula (Benth.) Epling and Lepechinia paniculata (Kunth) Epling. Plants 2019, 8, 1. [CrossRef] [PubMed]

46. Pragadheesh, V.S.; Saroj, A.; Yadav, A.; Samad, A.; Chanotiya, C.S. Compositions, enantiomer characterization and antifungal activity of two Ocimum essential oils. Ind. Crops Prod. 2013, 50, 333-337. [CrossRef]

47. Ramírez, J.; Gilardoni, G.; Ramón, E.; Tosi, S.; Picco, A.M.; Bicchi, C.; Vidari, G. Phytochemical study of the Ecuadorian species Lepechinia mutica (Benth.) Epling and high antifungal activity of carnosol against Pyricularia oryzae. Pharmaceuticals 2018, 11, 33. [CrossRef]

48. Coleman, W.M.; Lawrence, B.M. Examination of the enantiomeric distribution of certain monoterpene hydrocarbons in selected essential oils by automated solid-phase microextraction-chiral gas chromatography-mass selective detection. J. Chromatogr. Sci. 2000, 38, 95-99. [CrossRef]

49. Endeshaw, M.M.; Gautun, O.R.; Asfaw, N.; Aasen, A.J. Volatile oil constituents of the Ethiopian plant Salvia schimperi Benth. Flavour Fragr. J. 2000, 15, 27-30. [CrossRef]

50. Dein, M.; Munafo, J.P. Characterization of key odorants in hoary mountain mint, Pycnanthemum incanum. J. Agric. Food Chem. 2019, 67, 2589-2597. [CrossRef] [PubMed]

51. Satyal, P.; Pappas, R.S. Antique lavender essential oil from 1945, its chemical composition and enantiomeric distribution. Nat. Volatiles Essent. Oils 2016, 3, 20-25.

52. DeCarlo, A.; Johnson, S.; Okeke-Agulu, K.I.; Dosoky, N.S.; Wax, S.J.; Owolabi, M.S.; Setzer, W.N. Compositional analysis of the essential oil of Boswellia dalzielii frankincense from West Africa reveals two major chemotypes. Phytochemistry 2019, 164, 24-32. [CrossRef] [PubMed] 